\title{
Observation of High Transformer Ratio Plasma Wakefield Acceleration
}

\author{
Gregor Loisch, ${ }^{1,{ }^{*}}$ Galina Asova, ${ }^{1,2}$ Prach Boonpornprasert, ${ }^{1}$ Reinhard Brinkmann, ${ }^{3}$ Ye Chen, ${ }^{1}$ Johannes Engel, ${ }^{1}$ \\ James Good, ${ }^{1}$ Matthias Gross, ${ }^{1}$ Florian Grüner, ${ }^{4,5}$ Holger Huck, ${ }^{1}$ Davit Kalantaryan, ${ }^{1}$ \\ Mikhail Krasilnikov, ${ }^{1}$ Osip Lishilin, ${ }^{1}$ Alberto Martinez de la Ossa, ${ }^{4}$ Timon J. Mehrling, ${ }^{3,8}$ David Melkumyan, ${ }^{1}$ \\ Anne Oppelt, ${ }^{1}$ Jens Osterhoff, ${ }^{3}$ Houjun Qian, ${ }^{1}$ Yves Renier, ${ }^{1, \dagger}$ Frank Stephan, ${ }^{1}$ Carmen Tenholt, ${ }^{6}$ \\ Valentin Wohlfarth, ${ }^{1}$ and Quantang Zhao, ${ }^{1, \$}$ \\ ${ }^{1}$ Deutsches Elektronen-Synchrotron DESY, 15738 Zeuthen, Germany \\ ${ }^{2}$ INRNE, BAS, 1784 Sofia, Bulgaria \\ ${ }^{3}$ Deutsches Elektronen-Synchrotron DESY, 22607 Hamburg, Germany \\ ${ }^{4}$ Universität Hamburg, 22761 Hamburg, Germany \\ ${ }^{5}$ Center for Free-Electron Laser Science, 22607 Hamburg, Germany \\ ${ }^{6}$ CERN, 1211 Geneva, Switzerland
}

(Received 27 February 2018; published 7 August 2018)

\begin{abstract}
Particle-beam-driven plasma wakefield acceleration (PWFA) enables various novel high-gradient techniques for powering future compact light-source and high-energy physics applications. Here, a driving particle bunch excites a wakefield response in a plasma medium, which may rapidly accelerate a trailing witness beam. In this Letter, we present the measurement of ratios of acceleration of the witness bunch to deceleration of the driver bunch, the so-called transformer ratio, significantly exceeding the fundamental theoretical and thus far experimental limit of 2 in a PWFA. An electron bunch with ramped current profile was utilized to accelerate a witness bunch with a transformer ratio of $4.6_{-0.7}^{+2.2}$ in a plasma with length $\sim 10 \mathrm{~cm}$, also demonstrating stable transport of driver bunches with lengths on the order of the plasma wavelength.
\end{abstract}

DOI: 10.1103/PhysRevLett.121.064801

The demand for compact, high-energy electron accelerators for, e.g., x-ray free electron lasers or high-energy physics has given rise to greatly intensified research on alternative particle acceleration techniques that allow us to significantly shrink the accelerator lengths compared to conventional technology. One of the most promising candidates is the beam-driven plasma wakefield accelerator (PWFA) [1]. In this scheme, a relativistic particle bunch, the so-called driver, creates a wakefield in a plasma by altering the plasma electron distribution due to its space charge forces. A trailing, second (witness) bunch can be accelerated in this wakefield. Accelerating fields of tens of $\mathrm{GV} / \mathrm{m}$ have already been measured in PWFA experiments [2], exceeding the field strengths in conventional radiofrequency (rf) cavities by 3 orders of magnitude.

The PWFA concept allows for the transfer of energy from the driver bunch of average particle energy $E$ to the witness bunch through the plasma medium. The ratio between the maximum accelerating field in the witness

Published by the American Physical Society under the terms of the Creative Commons Attribution 4.0 International license. Further distribution of this work must maintain attribution to the author(s) and the published article's title, journal citation, and DOI. bunch and the maximum decelerating field in the driver bunch is called the transformer ratio $T_{R}[3,4]$, and energy gain of the witness bunch is limited to $\sim T_{R} E$.

Increasing the transformer ratio thus allows us to accelerate bunches to higher energies at constant driver bunch energy, or to reduce the length of the drive-beam accelerator, potentially reducing the size and cost of a PWFA facility significantly [5]. A higher transformer ratio can be achieved by flattening the decelerating wakefield along the driver bunch $[5,6]$. The flattened decelerating field also implies the maximization of the acceleration efficiency $[7,8]$.

For longitudinally symmetrical driver bunches, which typically emerge from conventional accelerators, the socalled fundamental theorem of beam-loading limits the transformer ratio in linear wakefield theory to values below or equal to 2 [3]. A transformer ratio above this limit is therefore considered high. Asymmetrical bunch shapes longer than the plasma wakefield wavelength $[5,6,9,10]$ as well as asymmetrical trains of bunchlets $[11,12]$ have been proposed to produce high transformer ratio (HTR) wakefields. These possibilities have been investigated theoretically, but thus far high transformer ratios have not been achieved in a PWFA. Only recently they were measured in dielectric-loaded structure-based wakefield accelerators [13-15]. 
The PWFA has demonstrated much higher acceleration gradients than other, structure-based wakefield accelerators, but it also inherently exhibits strong transverse wakefields. The instabilities resulting from these transverse fields for long, asymmetrical driver bunches were found to be a major physical challenge for HTR PWFA, as they inhibit the transport of HTR-capable driver bunches in the plasma medium over distances relevant for an accelerator [9,16-20]. This limitation was mitigated theoretically by operation in the nonlinear interaction regime [7,21-23], in which the driver bunch electron density locally exceeds the plasma electron density and thus all plasma electrons are expelled from the axis. In this regime, the transverse forces are predicted purely focusing along the driver bunch. The bunch shapes that are needed to increase the transformer ratio (i.e., flatten the decelerating field along the driver) in such a nonlinear wakefield were found to be similar to the linear regime, namely bunches with a linearly increasing current profile followed by a sharp cutoff at the tail and in some cases preceded by a precursor of various shapes [5,7,10,23]

In this Letter, we report on the demonstration of high transformer ratio plasma wakefield acceleration in the nonlinear regime by a ramped current profile, relativistic electron bunch, also validating the predictions of theoretical models for the mitigation of inhibiting plasma instabilities.

The experiments were carried out at the photoinjector test facility at DESY, Zeuthen site (PITZ). This rf, photoinjection electron accelerator operating at $1.3 \mathrm{GHz}$ is capable of generating electron bunches with charges of $0.01-5 \mathrm{nC}$ at energies up to $25 \mathrm{MeV}$ and normalized emittances as low as $0.1 \mathrm{~mm} \mathrm{mrad}$ [24]. The ramped electron bunch profile is produced by shaping the photocathode-laser temporal profile, which translates into the electron bunch temporal profile $[25,26]$. In the PITZ laser pulse shaper the initial $0.7 \mathrm{ps}$ long (rms) Gaussian laser pulse is split into 14 quasipulses that are individually delayed and coherently added to form a longitudinal laser profile of tunable shape and a fixed length of 20 ps (measured as the delay between first and last Gaussian quasipulse). The witness bunch is created by splitting the photocathode-laser pulse before it enters the pulse shaper and merging the beam paths again before being sent to the photocathode. The delay between driver and witness is adjusted by changing the relative path length of main to witness laser pulse. Charge ratio and total charge are controlled independently. To diagnose the longitudinal electron bunch profile, a $2.997 \mathrm{GHz}$ transverse deflecting structure, operated at zero-crossing phase, in the high-energy section of the PITZ beam line is used [27,28], as shown in Fig. 1. The time-dependently deflected electrons of the bunch are imaged on a ceriumdoped yttrium aluminum garnet (Ce:YAG) scintillator with a charge coupled device (CCD) camera.

The measured and simulated electron bunch current profiles are shown in Fig. 2. For the measurements presented

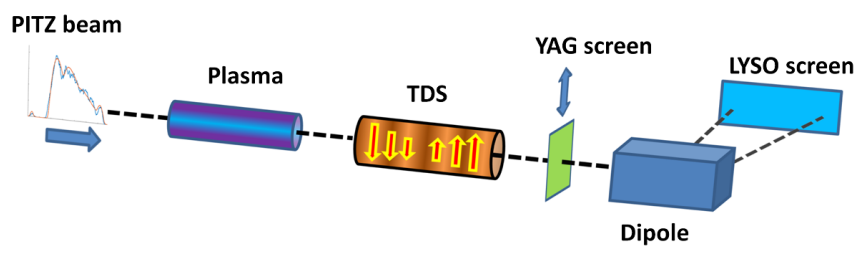

FIG. 1. Schematic layout of the measurement setup with the plasma, the transverse deflecting structure (TDS), the movable YAG screen for bunch profile measurements, the $60^{\circ}$ sector dipole spectrometer, and the LYSO screen for longitudinal phase space measurements.

here the driver bunch charge was $(508 \pm 10) \mathrm{pC}$, the witness bunch charge $(10 \pm 6) \mathrm{pC}$. Their lengths corresponded to the laser pulse lengths of about 20 ps (driver, as defined before) and 0.7 ps (witness, rms), respectively. The delay between the current maxima of the two bunches was $10 \mathrm{ps}$. The total charge of $(518 \pm 16) \mathrm{pC}$ was measured using a Faraday cup at the photoelectron-gun exit and the charge ratio between driver and witness bunches was determined from the intensity of their scintillation light signals on the Ce:YAG screen.

Upstream of the transverse deflecting structure a gas discharge plasma cell was inserted into the beam line. It consisted of a $100 \mathrm{~mm}$ long gas cell with an electrode at each end. The 0.6 mbar argon gas within the cell was separated by $2 \mu \mathrm{m}$ thick, metalized polymer foil windows from the ultrahigh vacuum of the accelerator beam line. A $2.4 \mathrm{kV}, 250 \mathrm{~A}$ discharge pulse of $2 \mu$ s length was applied between the electrodes which leads to plasma formation in the cell gas. The plasma density is measured from the periodicity of longitudinal phase space modulations introduced to a longitudinally flattop-shaped electron bunch by the plasma via the self-modulation instability in a separate

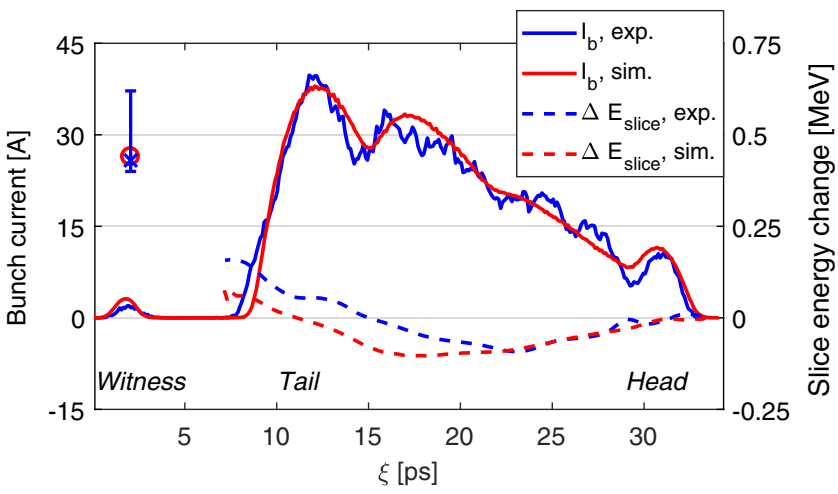

FIG. 2. Experimental and simulated electron bunch currents $I_{b}$ (solid lines) and slice energy changes $\Delta E_{\text {slice }}$ (dashed lines) between plasma off and on cases in the comoving coordinate $\xi=z / c$. Blue cross and red circle indicate measured and simulated maximum witness energy gain, respectively. Total charge of ramped driver (right) and short, low charge Gaussian witness bunch (left) is $518 \pm 16 \mathrm{pC}$. The time resolution of the measurement is $0.6 \mathrm{ps}$. 
(a)

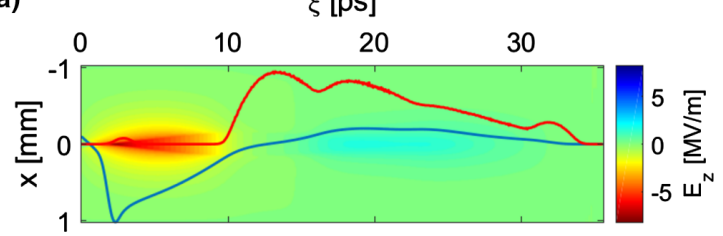

(b)

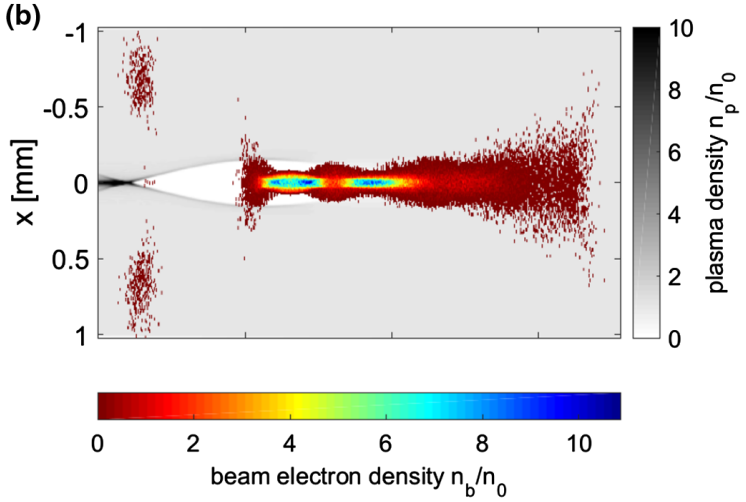

FIG. 3. (a) Simulated longitudinal electrical field distribution and (b) beam electron (color scale) and plasma electron (gray scale) densities after $64 \mathrm{~mm}$ of propagation into the $2.2 \times$ $10^{13} \mathrm{~cm}^{-3}$ plasma. The red line in (a) indicates the bunch current profile which peaks at $38 \mathrm{~A}$. The blue line shows the longitudinal electrical field on axis and shares the axis of the color map.

measurement [29]. The maximum density of $5 \times 10^{15} \mathrm{~cm}^{-3}$, observed just after the end of the current pulse, decays to $10^{13} \mathrm{~cm}^{-3}$ in about $300 \mu \mathrm{s}$. By changing the delay between plasma ignition and the electron beam arrival, the plasma density at the time of interaction could be adjusted.

For the simulations of the presented measurements, $10^{6}$ macroparticles, representing the measured electron bunches, were tracked through the accelerator beam line using ASTRA [30] and then imported into the 3D particle in cell (PIC) code HIPACE [31], in which the beam-plasma interaction was modeled. A grid of $512 \times 256 \times 256$ cells was used in HIPACE to simulate a comoving frame of $10.65 \times$ $1.13 \times 1.13 \mathrm{~mm}^{3}$ volume. The box length in the direction of beam movement corresponds to $35.51 \mathrm{ps}$ in the comoving coordinate $\xi=z / c$. Figure 3 shows the $x-z$ plane next to the $y$ axis of the described HIPACE simulation volume at a plasma density of $2.2 \times 10^{13} \mathrm{~cm}^{-3}$. In Fig. 3(a) the shape of the longitudinal bunch profile (red line, see also Fig. 2) and the electrical field profile (blue line) on axis underlayed with the 2D longitudinal field distribution near axis are depicted. The on-axis field is clearly nonlinearly shaped in the back of the plasma bubble. The beam and plasma electron density distributions near the axis are shown in Fig. 3(b). Driver bunch electron densities significantly exceed the unperturbed plasma electron density $n_{0}$, clearly setting the interaction in the nonlinear regime as required for mitigation of beamplasma instabilities $[7,20,23]$.

As shown on the left side of Fig. 3(b), only a fraction of the witness bunch electrons is trapped and accelerated in the plasma wake over the full plasma length due to the witness bunch not being focused at the plasma entrance. This is caused by the different charge densities of driver and witness at the photocathode, which result in different Twiss parameters [32,33] for both bunches. Transport is thus only optimized for the driver bunch in order to reach highest bunch charge density in the plasma for a nonlinear PWFA interaction. This effect limits the trapping efficiency for the witness to $30 \%$ in this experiment and causes a fraction of the witness beam being defocused by the wakefield, which is then partially lost on apertures in the subsequent beam line.

In the experiment, the longitudinal phase space of the bunches was measured by using the transverse (vertically) deflecting cavity and a $60^{\circ}$, horizontal dipole spectrometer, as sketched in Fig. 1. From the longitudinal phase space the energy gain of the witness bunch and the slice energy loss of the driving bunch are determined. The transformer ratios are calculated from these values as described below. This method yields an effective transformer ratio, averaged over the full acceleration length. Measurements of the longitudinal phase spaces without and with plasma interaction are shown in Figs. 4(a) and 4(b), respectively. Owing to the large difference in charge between driver and witness bunches they are imaged on a cerium-doped lutetium yttrium orthosilicate scintillator (LYSO) screen using the same CCD camera but with different CCD gains ( 0 vs $18 \mathrm{~dB}$ without, 6 vs $22 \mathrm{~dB}$ with plasma interaction) for improved dynamic range. The change in driver mean slice energy [Figs. 4(a) and 4(b), white dashed lines] with and without plasma interaction is shown along the bunch current profile in Fig. 2.

The transformer ratio is calculated in two different ways:

(i) The gain of the mean energy of the witness bunch (Fig. 4, green dashed lines) is divided by the highest mean slice energy loss (Fig. 2, blue dashed line) in the driver bunch.

(ii) The gain of the high-energy tail of the witness bunch (Fig. 4, red dashed lines) is divided by the highest mean slice energy loss (Fig. 2, blue dashed line) in the driver bunch.

Method (i) results in a transformer ratio for the full witness bunch, which would be relevant for the suitability of the setup as a plasma wakefield acceleration stage. Method (ii) represents the maximum transformer ratio in the excited wakefield, i.e., the optimum ratio that can be reached for a witness bunch by proper transverse and phase matching of the witness to the driven wake. As mentioned above, the electron beam optics for driver and witness bunch in the presented experiment were identical, whereas their space charge density significantly differed. This led to the low trapping efficiency seen in the simulations and could be overcome by separate production and transport of driver and witness beam, as it will be necessary for staged wakefield accelerators. 
(a) without plasma

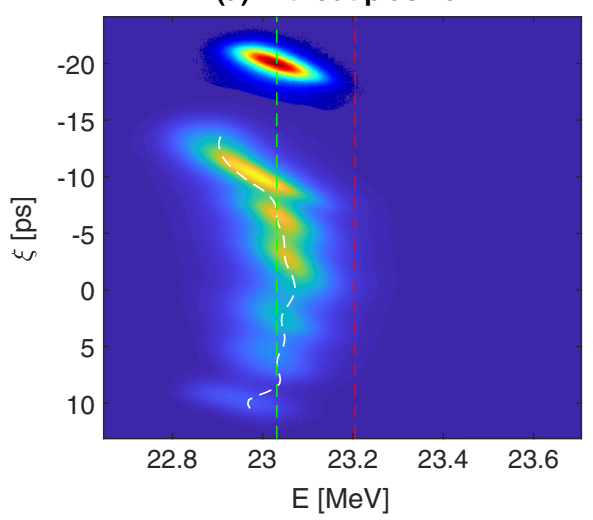

(b) with plasma

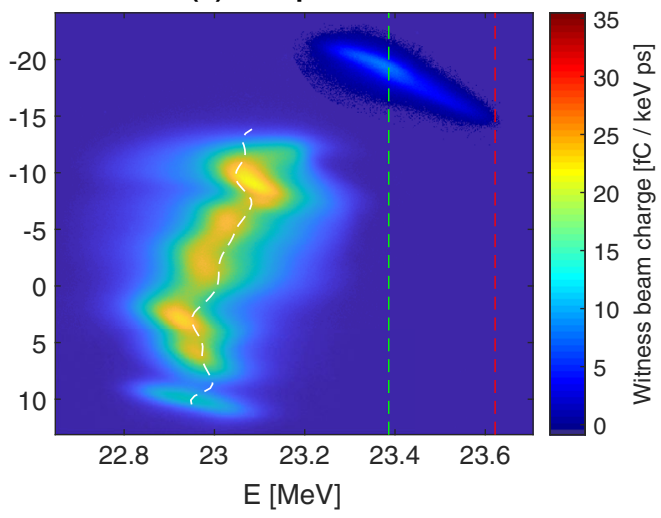

FIG. 4. Longitudinal phase space of driver and witness bunches measured without (a) and with (b) plasma acceleration in a plasma of $2 \times 10^{13} \mathrm{~cm}^{-3}$ electron density. Note that the upper (witness) bunch was imaged with a different camera gain than the driver. Dashed lines indicate the witness mean (green) and maximum (red) energy and the driver mean slice energies (white). The bunch current and the measured slice energy changes inside of the driver bunch, corresponding to this measurement, are shown in Fig. 2.

The mean energy gain of the witness bunch for a plasma density of $(2.0 \pm 0.3) \times 10^{13} \mathrm{~cm}^{-3}$ was measured to be $(0.36 \pm 0.01) \mathrm{MeV}$ and the energy gain of the highenergy tail of the witness bunch was measured to be $\left(0.43_{-0.02}^{+0.19}\right) \mathrm{MeV}$, corresponding to transformer ratios of $3.9 \pm 0.7$ and $4.6_{-0.7}^{+2.2}$ for methods (i) and (ii), respectively, at a maximum slice energy loss of the driver bunch of $(0.09 \pm 0.01) \mathrm{MeV}$.

The given errors include the standard deviation of the slice energy changes in the driver bunch and the standard deviation of the witness bunch mean [method (i)] or maximum [method (ii)] energy for ten consecutive measurements. Furthermore, the influence of the transverse bunch size of the witness bunch, measured on a reference screen, on the measured maximum witness energies and systematic measurement uncertainties such as screen resolution are included. To account for the fact that the highest energy electrons after acceleration in the plasma are not necessarily at the highest energies before the acceleration, the positive error in method (ii) includes the difference between mean and maximum energy of the witness bunch without plasma.

Because of the low maximum current of the driver bunch of $\leq 40 \mathrm{~A}$, the maximum wakefield amplitudes of a few $\mathrm{MV} / \mathrm{m}$ were not nearly as high as has been reported from other PWFA experiments. Nevertheless, the shape of the energy loss along the bunch, i.e., the integrated wakefield is clearly resolvable in Fig. 2 . The wakefield amplitude is not flat along the bunch, as predicted for an ideally matched case [7]. The reason for this could be nonideal focusing of the driver into the plasma or a mismatch of the bunch shape, e.g., the nonideal slope of the linearly rising part of the driver current.

In summary, we have shown that a $20 \mathrm{ps,} \mathrm{ramped}$ current profile bunch, created by photocathode-laser based bunch shaping, excited a nonlinear wakefield in a $(2 \pm 0.3) \times 10^{13} \mathrm{~cm}^{-3}$ argon gas discharge plasma with a transformer ratio of $4.6_{-0.7}^{+2.2}$. This measurement first demonstrates the feasibility of high transformer ratios in a plasma wakefield accelerator. The results also validate the predictions of the theoretical concept to operate a HTR PWFA in the nonlinear regime for mitigating beamplasma instabilities, which would inhibit the transport of HTR-capable driver bunches. Neither the transverse two stream or self-modulation instability $[9,16,18,34]$ nor the hose instability $[17,19]$ have been observed in the HTR acceleration over multiple instability growth lengths (SMI 13, hosing 10) [18], as it is required for PWFA with considerable energy gain. The presented transformer ratio enables plasma wakefield acceleration by 2.3 times higher energies or a corresponding reduction of the driver bunch acceleration length, compared to acceleration with the transformer ratio limit imposed by the fundamental theorem of beam loading. Applying the methods developed in this study at high-energy PWFA facilities by modification of the photocathode laser would allow scaling our results to the $\mathrm{GeV}$ energy gain range, utilizing the higher energy beams and bunch compression tools available in such facilities.

These results are a crucial step towards reaching high energies in compact, beam driven plasma accelerators and maximizing the efficiency for future high-energy colliders. Further steps will focus on improving the bunch shaping, for increase of transformer ratios and wakefield flatness, and optimizing the bunch shaping flexibility for highenergy experiments.

The authors would like to thank A. Yeremyan and A. Vardanyan for their participation in the measurement campaign as well as other colleagues from CANDLE, Yerevan, Armenia for their great support in operating the PITZ facility. 
*gregor.loisch@desy.de

${ }^{\dagger}$ Present address: DPNC-Universitè de Genève, 1211 Geneva, Switzerland.

${ }^{\ddagger}$ On leave from Institute of Modern Physics, 730000 Lanzhou, China.

${ }^{\S}$ Present address: Lawrence Berkeley National Laboratory, University of California, Berkeley, California 94720, USA.

[1] P. Chen, J. M. Dawson, R. W. Huff, and T. Katsouleas, Acceleration of electrons by the interaction of a bunched electron beam with a plasma, Phys. Rev. Lett. 54, 693 (1985).

[2] I. Blumenfeld, C. E. Clayton, F.-J. Decker, M. J. Hogan, C. Huang, R. Ischebeck, R. Iverson, C. Joshi, T. Katsouleas, N. Kirby, W. Lu, K. A. Marsh, W. B. Mori, P. Muggli, E. Öz, R. H. Siemann, D. Walz, and M. Zhou, Energy doubling of $42 \mathrm{GeV}$ electrons in a metre-scale plasma wakefield accelerator, Nature (London) 445, 741 (2007).

[3] K. L. F. Bane, P. B. Wilson, and T. Weiland, Wake fields and wake field acceleration, Stanford Linear Accelerator Center Report No. SLAC-PUB-3528, 1984; also in AIP Conf. Proc. Phys. High Energy Accel. 127, 875 (1984).

[4] R. D. Ruth, A. W. Chao, P. L. Morton, and P. B. Wilson, A plasma wake field accelerator, Stanford Linear Accelerator Center Report No. SLAC-PUB-3374, 1984.

[5] P. Chen, J. J. Su, J. M. Dawson, K. L. F. Bane, and P. B. Wilson, Energy Transfer in the Plasma Wake-Field Accelerator, Phys. Rev. Lett. 56, 1252 (1986).

[6] K. L. F. Bane, P. Chen, and P. Wilson, On collinear wake field acceleration, IEEE Trans. Nucl. Sci. 32, 3524 (1985).

[7] K. V. Lotov, Efficient operating mode of the plasma wakefield accelerator, Phys. Plasmas 12, 053105 (2005).

[8] M. Litos, E. Adli, W. An, C. I. Clarke, C. E. Clayton, S. Corde, J. P. Delahaye, R. J. England, A. S. Fisher, J. Frederico, S. Gessner, S. Z. Green, M. J. Hogan, C. Joshi, W. Lu, K. A. Marsh, W. B. Mori, P. Muggli, N. VafaeiNajafabadi, D. Walz et al., High-efficiency acceleration of an electron beam in a plasma wakefield accelerator, Nature (London) 515, 92 (2014).

[9] T. Katsouleas, Physical mechanisms in the plasma wakefield accelerator, Phys. Rev. A 33, 2056 (1986).

[10] B. Jiang, C. Jing, P. Schoessow, J. Power, and W. Gai, Formation of a novel shaped bunch to enhance transformer ratio in collinear wakefield accelerators, Phys. Rev. ST Accel. Beams 15, 011301 (2012).

[11] E. Laziev, V. Tsakanov, and S. Vahanyan, Electromagnetic wave generation with high transformation ratio by intense charged particle bunches, in Proceedings of the 1st European Particle Accelerator Conference (EPAC 88), Rome, Italy, 1988, Vol. 1040 (World Scientific, Singapore, 1989); IAEA INIS Report No. EFI 1040 (3), 1988.

[12] J. P. Farmer, R. Martorelli, and A. Pukhov, Transformer ratio saturation in a beam-driven wakefield accelerator, Phys. Plasmas 22, 123113 (2015).

[13] C. Jing, A. Kanareykin, J. G. Power, M. Conde, Z. Yusof, P. Schoessow, and W. Gai, Observation of Enhanced Transformer Ratio in Collinear Wakefield Acceleration, Phys. Rev. Lett. 98, 144801 (2007).

[14] C. Jing, J. G. Power, M. Conde, W. Liu, Z. Yusof, A. Kanareykin, and W. Gai, Increasing the transformer ratio at the Argonne wakefield accelerator, Phys. Rev. ST Accel. Beams 14, 021302 (2011).

[15] Q. Gao, G. Ha, C. Jing, S. P. Antipov, J. G. Power, M. Conde, W. Gai, H. Chen, J. Shi, E. E. Wisniewski, D. S. Doran, W. Liu, C. E. Whiteford, A. Zholents, P. Piot, and S. S. Baturin, Observation of High Transformer Ratio of Shaped Bunch Generated by an Emittance-Exchange Beam Line, Phys. Rev. Lett. 120, 114801 (2018).

[16] K. V. Lotov, Simulation of ultrarelativistic beam dynamics in the plasma wake-field accelerator, Nucl. Instrum. Methods Phys. Res., Sect. A 410, 461 (1998).

[17] D. H. Whittum, W. M. Sharp, S. S. Yu, M. Lampe, and G. Joyce, Electron-Hose Instability in the Ion-Focused Regime, Phys. Rev. Lett. 67, 991 (1991).

[18] C. B. Schroeder, C. Benedetti, E. Esarey, F. J. Grüner, and W. P. Leemans, Growth and phase velocity of self-modulated beam-driven plasma waves, Phy. Rev. Lett. 107, 145002 (2011).

[19] T. J. Mehrling, R. A. Fonseca, A. Martinez de la Ossa, and J. Vieira, Mitigation of the hose instability in plasma-wakefield accelerators, Phy. Rev. Lett. 118, 174801 (2017).

[20] J. Krall and G. Joyce, Transverse equilibrium and stability of the primary beam in the plasma wake-field accelerator, Phys. Plasmas 2, 1326 (1995).

[21] J. B. Rosenzweig, Nonlinear Plasma Dynamics in the Plasma Wake-Field Accelerator, Phys. Rev. Lett. 58, 555 (1987).

[22] J. B. Rosenzweig, N. Barov, M. C. Thompson, and R. B. Yoder, Energy loss of a high charge bunched electron beam in plasma: Simulations, scaling, and accelerating wakefields, Phys. Rev. ST Accel. Beams 7, 061302 (2004).

[23] J. B. Rosenzweig, B. Breizman, T. Katsouleas, and J. J. Su, Acceleration and focusing of electrons in two-dimensional nonlinear plasma wake fields, Phys. Rev. A 44, R6189 (1991).

[24] M. Krasilnikov, F. Stephan, G. Asova, H.-J. Grabosch, M. Gross, L. Hakobyan, I. Isaev, Y. Ivanisenko, L. Jachmann, M. Khojoyan, G. Klemz, W. Köhler, M. Mahgoub, D. Malyutin, M. Nozdrin, A. Oppelt, M. Otevrel, B. Petrosyan, S. Rimjaem, A. Shapovalov et al., Experimentally minimized beam emittance from and L-band photoinjector, Phys. Rev. ST Accel. Beams 15, 100701 (2012).

[25] I. Will and G. Klemz, Generation of flat-top picosecond pulses by coherent pulse stacking in a multicrystal birefringent filter, Opt. Express 16, 14922 (2008).

[26] G. Loisch, R. Brinkmann, J. Good, M. Gross, F. Grüner, H. Huck, I. Isaev, M. Krasilnikov, O. Lishilin, A. Oppelt, Y. Renier, F. Stephan, and I. Will, Photocathode laser based bunch shaping for high transformer ratio plasma wakefield acceleration, Nucl. Instrum. Methods Phys. Res. A (to be published), https://www.sciencedirect.com/science/article/ pii/S0168900218301979.

[27] L. Kravchuk, A. Anisimov, D. Churanov, A. Donat, C. Gerth, M. Hoffmann, M. Huening, E. Ivanov, W. Koehler, M. Krasilnikov, S. Kutsaev, M. Lalayan, J. Meissner, V. Paramonov, M. Pohl, J. Schultze, A. Smirnov, N. Sobenin, F. Stephan, G. Trowitzsch et al., Layout of the PITZ transverse deflecting system for longitudinal phase space and slice emittance measurements, in Proceedings of the 
XXVth Linear Accelerator Conference, Tokyo, 2010, Report No. TUP011 (JACoW, Geneva, Switzerland, 2011).

[28] H. Huck, G. Asova, M. Bakr, P. Boonpornprasert, A. Donat, J. Good, M. Gross, C. Hernandez-Garcia, I. Isaev, L. Jachmann, D. Kalantaryan, M. Khojoyan, W. Koehler, G. Kourkafas, M. Krasilnikov, D. Malyutin, D. Melkumyan, A. Oppelt, M. Otevrel, M. Pohl et al., First results of commissioning of the PITZ transverse deflecting structure, in Proceedings of the 37th International Free Electron Laser Conference (FEL 2015), Daejeon, Korea, 2015, Report No. MOP039 (JACoW, Geneva, Switzerland, 2015).

[29] G. Loisch et al., Plasma density measurements by means of self-modulation of long electron bunches (to be published).

[30] K. Flöttmann, Astra-A space charge tracking algorithm, http://www.desy.de/ mpyflo/ (2017).
[31] T. Mehrling, C. Benedetti, C. B. Schroeder, and J. Osterhoff, HiPACE: A quasi-static particle-in-cell code, Plasma Phys. Controlled Fusion 56, 084012 (2014).

[32] R. Q. Twiss and N. H. Frank, Orbital stability in a proton synchrotron, Rev. Sci. Instrum. 20, 1 (1949).

[33] E. D. Courant and H. S. Snyder, Theory of the alternatinggradient synchrotron, Ann. Phys. (N.Y.) 3, 1 (1958).

[34] M. Gross, J. Engel, J. Good, I. Isaev, G. Koss, M. Krasilnikov, O. Lishilin, G. Loisch, Y. Renier, T. Rublack, F. Stephan, R. Brinkmann, A. Martinez de la Ossa, J. Osterhoff, D. Malyutin, D. Richter, T. Mehrling, M. Khojoyan, C. B. Schroeder, and F. Grüner, Observation of the Self-Modulation Instability via Time-Resolved Measurements, Phys. Rev. Lett. 120, 144802 (2018). 